\title{
VALEUR RELATIVE DES EXAMENS DANS LA MAMIMITE CHRONIQUE DES BOVIDÉS
}

\author{
par \\ G. J. HUCKER et Poul ARne HANSEN (1)
}

(New York State Agricultural Experiment Station Geneva, New York, U.S.A.).

On a constaté, pendant ces dernières années, une tendance à attacher de plus en plus d'importance à la mammite. Ceci est vrai, non seulement de la part des fonctionnaires du Contrôle de l'Hygiène publique, mais aussi de celle des personnes intéressées à l'économie laitière. Tous admettent que les infections de la mamelle constituent un problème important. Le rapport possible entre les maux de gorge septiques épidémiques et les infections de la mamelle ont attiré l'attention des fonctionnaires de l'Hygiène publique. Toutefois, en laissant de côté cette partie du problème, et en ne se plaçant strictement qu'au point de vue de l'étiologie des maladies humaines, la mammite n'a vraisemblablement que peu d'importance en ce qui concerne l'hygiène sociale.

L'emploi des termes relatifs aux infections de la mamelle est devenu quelque peu confus. Le terme "mammite" a été appliqué d'une façon générale à tous les processus pathologiques de la mamelle. Pour la clarté de ce qui va suivre, les termes utilisés à propos des infections de la mamelle vont être définis. Pour débuter, il doit être entendu que l'étude de la mammite ne comprend. pas nécessairement le problème des maux de gorge septiques épidémiques dus au lait. Les mamelles qui renferment les organismes auxquels sont dues ces maladies épidémiques, ont probablement été infectées par une source humaine avec un type de streptocoque distinct de celui trouvé dans la forme habituelle de la mammite streptococcique. Les mamelles infectées par le streptocoque du type "mastitis" peuvent n'être jamais cause d'un mal de gorge septique épidémique. En d'autres termes, les mamelles infectées avec un streptocoque de type humain peuvent produire le même processus pathologique et clinique dans la mamelle qu'une infection (mammite) causée par les streptocoques bovins plus communs. La présente étude ne s'occupe pas des mamelles infectées par le streptocoque de type humain.

$\mathrm{Du}$ point de vue du clinicien, il semble y avoir deux types distincts de mammite streptococeique. Le type aigu ou clinique est relativement rare. Dans ce cas, les symptômes cliniques comprennent $\mathrm{p}$ เrfois la toxémie, une mamelle enflée et très fortement enflammée, avec prostation de l'individu, tandis qu'on ne peut obtenir des quartiers infectés que peu ou pas de lait. Il y a habituellement pro-

(1) Traduction de C. Wotf. 
duction de pus. On note une élévation sensible de la température en certains cas. Il n'est pas nécessaire d'employer des méthodes spéciales pour déceler ce type de mammite, car l'état du sujet est évident.

Le type sous-clinique, plus commun, souvent appelé mammite chronique ou latente, contraste avec la mammite clinique ou aiguë. Dans ce cas, le sujet peut paraître pratiquement normal. Il n'y a pas de toxémie visible. On ne note que peu ou pas d'élévation de la température, et la mamelle peut ne pas être enflée ou enflammée. Le lait peut être normal en apparence quand on le filtre sur une gaze ou un tamis fin. Dans les cas graves, on peut trouver du pus. Dans certains cas, on peut aussi noter un aspect "aqueux» du lait. Le sujet paraît être suffisamment normal pour rester soumis à la traite. L'infection peut se manifester à intervalles irréguliers par des symptômes partiellement aigus. Dans de telles conditions, le sujet est considéré comme ayant des "accès soudains " ou de la mammite aiguë quand il est soumis à des conditions anormales, telles que le froid, une nourriture riche en protéine, une traite incomplète, etc.

Les types cliniques et chroniques peuvent quelquefois être rapidement tansmis dans le troupeau, ou bien ils peuvent apparaître sous forme de cas sporadiques isolés.

Il faut aussi noter que quand les cliniciens discutent les infections de la mamelle, ils peuvent employer une terminologie dans laquelle les termes "fibrose " et "mammite " sont interchangeables. Dans de telles conditions, la constatation d'une "mammite nette" peut ne signifier que la présence d'une forte proportion de tissu cicatriciel ou de fibrose sans qu'jl soit tenu compte de la présence ou de l'absence d'infections bactériennes.

L'incidence et la découverte de mammites sous-cliniques ou chroniques, ou de ces cas montrant une infection de la mamelle par des streptocoques de classe inférieure, servent de base à l'étude actuelle. Un grand nombre de vaches qui servirent à donner des renseignements pour cette étude purent être autopsiées, et les résultats furent utilisés comme base pour juger de la valeur des divers essais. Jusqu'à présent, les différentes méthodes ont été étudiées en les comparant entre elles et les résultats ainsi obtenus n'étaient que relatifs. Dans la présente recherche, la présence ou l'absence d'une condition pathologique de la mamelle a été réellement déterminée par l'examen post-mortem. Toutes les vaches montrant de l'induration ou de la fibrose à l'autopsie étaient considérées comme ayant subi, à un moment donné de leur vie, une infection ayant donné naissance à une mammite du type clinique ou du type sous-clinique. Il est probable que c'est la dernière qui est en cause, d'autant plus que ces vaches restèrent soumises à la traite dans leurs troupeaux 
respectifs jusqu'à ce qu'elles en fussent enlevées pendant la présente recherche. En d'autres termes, toutes les vaches montrant des indurations ou du tissu cicatriciel au cours de cette étude furent considérées comme ayant ou ayant eu de la mammite. Comme ce tissu cicatriciel n'était pas accompagné d'inflammation ou d'irrégularités manifestes du lait ou de la mamelle, la mammite fut considérée comme sous-clinique.

Il faut se rappeler que l'adoption de la présence de tissu cicatriciel dans la mamelle comme base de comparaison de la valeur des divers essais de laboratoire n'implique pas nécessairement que les auteurs admettent que sa présence signifie toujours une mammite ancienne ou actuelle. L'interprétation employée ici est simplement adoptée comme un indicateur utilisé pour juger les divers essais de laboratoire. D'autres études pour rechercher la véritable signification bactériologique des indurations de la mamelle sont en cours.

On a utilisé comme base, pour juger de la valeur des essais étudiés, la comparaison des résultats d'une étude bactériologique et clinique du lait obtenu de chaque quartier des vaches avant l'autopsie ainsi que les résultats de l'autopsie. En outre, on tint aussi compte de la fréquence relative avec laquelle des réactions positives furent obtenues par les divers essais pour évaluer la valeur des procédés étudiés.

On a supposé que toutes les modifications pathologiques trouvées dans les mamelles étudiées à l'autopsie étaient dues à des infections streptococciques. Dans aucun des cas, on n'a trouvé de traces de lésions tuberculeuses. Les résultats notés pour six vaches montrant une infection possible avec le bacillus coli furent éliminés de la présente étude.

\section{TESTS POUR LA MAMMITE CHRONIQUE.}

Le type chronique ou sous-clinique de mammite est celui rencontré le plus souvent et il peut être difficile à déceler. Le type aigu n'exige pas de méthode de recherche aussi soigneusement établie, car l'état est net. On trouve habituellement que le type de mammite à mal de gorge septique est le résultat d'épidémies de maux de gorge septiques et il peut être si peu aigu qu'il soit nécessaire d'utiliser les tests habituels décrits ci-dessous pour isoler la vache cause de difficulté. Toutefois, à ce sujet, des méthodes précises pour reconnaître définitivement les streptocoques hémolytiques impliqués dans ce type particulier d'infection ont été étudiées.

MODE D'OBTENTION DES ÉCHANTILLONS. - Les tests décrits ci-dessous peuvent être utilisés sur les échantillons de n'importe quel type. Toutefois, si l'on désire des renseignements plus précis, il faut prélever directement de la mamelle des échantillons frais pour 


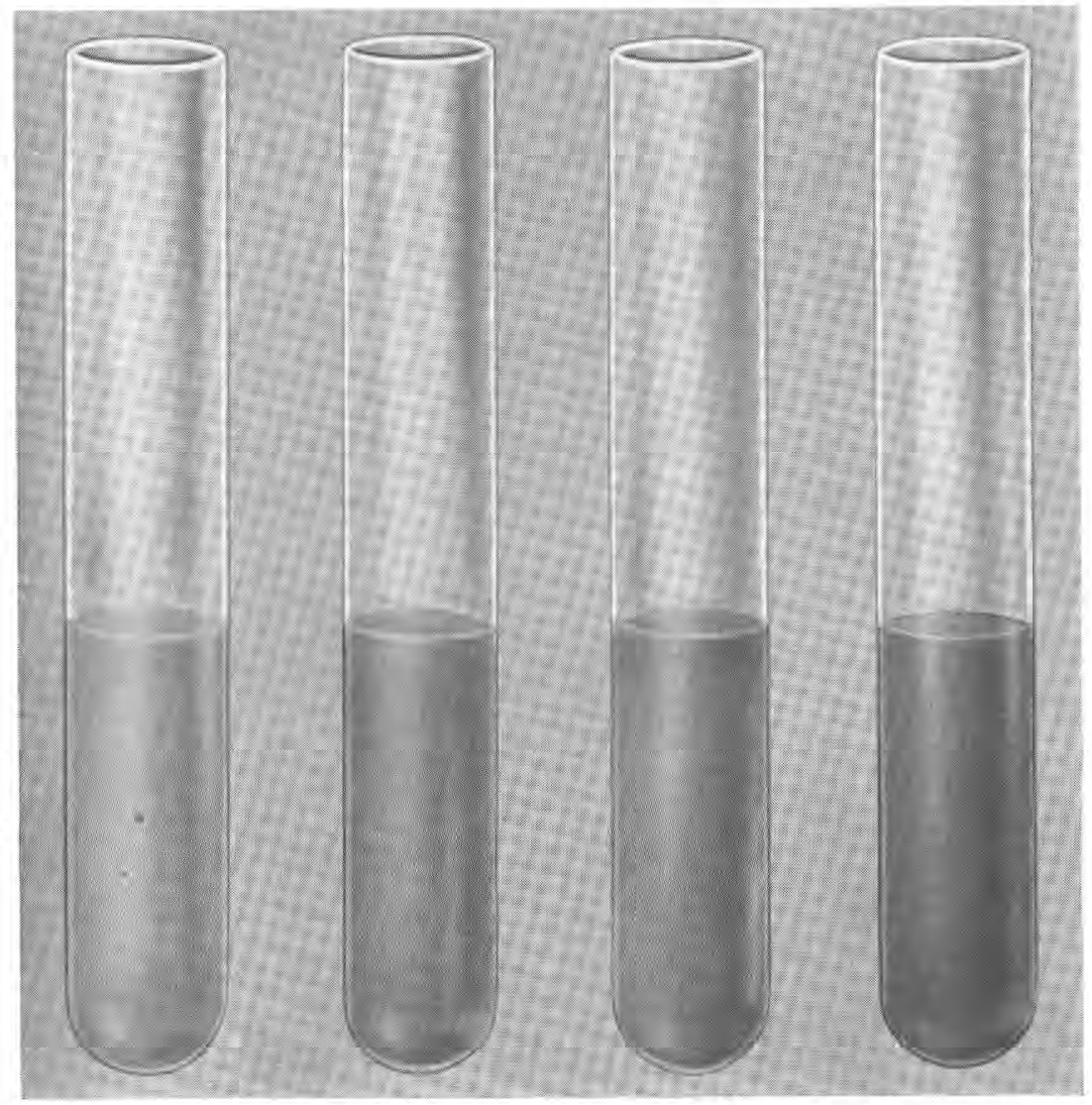

a.

b

c

d

Figure 1 - Essai au bleu de bromo-thymol appliqué au lait fraîchement trait.

a) Lait plus acide $(\mathrm{pH}: 6,2)$ que la normale.

b) Lait normal ( $\mathrm{pH}: 6,5)$.

c) Lait légèrement alcalin $(\mathrm{pH}: 6,9)$ suspect de mammite

d) Lait alcalin $(\mathrm{pH}: 7,0+)$ d'un pis atteint de mammite.

Cette planche appartient au travail de M. HUCKER qui a bien voulu nous l'adresser en faisant faire un nouveau tirage pour la Revue Le Lait. 
examen au laboratoire. La mamelle devra être nettoyée et quatre ou cinq jets de lait tirés avant que l'échantillon ne soit prélevé.

Il y a une différence considérable dans les résultats obtenus avec le premier lait et le dernier. On a trouvé par expérience que les résultats sont plus constants si l'échantillon est obtenu en recueillant le lait après qu'un nombre suffisant de jets de premier lait ont été rejetés. Les méthodes suivantes peuvent être employées pour rechercher les infections streptococeiques de la mamelle.

TEST AU BLEU DE BROMOTHYMOL (THYBROMOL).

Méthode. - Dans la méthode du tube à essai (1), 4 gouttes d'une solution concentrée de bleu de bromothymol sont ajoutées à $3 \mathrm{~cm}^{3}$ de lait. La solution de bleu de bromothymol peut être préparée en dissolvant 2 grammes de bleu de bromothymol dans $350 \mathrm{~cm}^{3}$ d'alcool.

Le lait devra être fraîchement tiré, et l'indicateur bien mélangé avec l'échantillon avant qu'on ne procède à la lecture. Il faudra examiner des échantillons de chaque quartier, et on pourra examiner les quatre tubes ensemble après addition de l'indicateur. Un quartier donnant du lait alcalin peut être rapidement décelé par ce procédé en comparant la couleur des échantillons examinés. Les quartiers infectés donneront, à l'addition de la solution indicatrice, une couleur bleue plus sombre que les quartiers normaux. Le lait normal est jaune verdâtre, du lait légèrement infecté est vert clair, et du lait infecté, de blèu verdâtre à bleu. Dans certains cas rares, le lait anormal peut être jaune vif. Pour des comparaisons plus précises, se reporter à la table des colorations de la planche 1 .

Dans la méthode du papier, on peut se procurer un étui de papier au bleu de bromothymol et on prélève une goutte de lait à l'extrémité du pis avec un petit morceau de ce papier. La coloration bleue qui se montre dans ce cas est analogue à celle obtenue dans le tube à essai. La méthode est plus économique et plus facile à exécuter que le procédé du tube à essai.

Utilisation du test. - L'essai au bleu de bromothymol est employé par beaucoup de vétérinaires en même temps qu'un examen de la mamelle et le bol d'essai de traite pour constituer un examen appelé " physique". Ce test seul est excellent à la ferme pour découvrir rapidement les vaches suspectes et infectées. S'il est employé régulièrement par le laitier à la ferme, des essais sur tous les quartiers du troupeau devraient être effectués au moins une fois chaque semaine.

(1) Les produits pour cet essai peuvent être obtenus de "The La Motte Chenical Produets Company, McCormick Building, Baltimore, Md. (U.S.A.) " ou de "L. F. Nafis Company, 23 North Desplaines St., Chicago, Ill. (U.S.A.) ". 
Culture quantitative en tubes inclinés d'agar de Burri. - BurRI (1) a inventé une méthode (fig. 2) pour l'examen bactériologique du lait usant des tubes inclinés d'agar. Une boucle de dimensions exactes remplace des pipettes pour mesurer le lait.

Mode opératoire. - Des tubes inclinés d'agar sont préparés en employant le milieu suivant :

Botillon de veau (2) $\ldots \ldots \ldots \ldots \ldots \ldots \ldots \ldots \ldots, 1.000 \mathrm{em}^{3}$

Agar Bacto . . . . . .

Glucose ....................... $2 \mathrm{gr.} 5$

Réaction amenée au $p$ H 7,2 .

Les tubes d'agar sont placés en position oblique pendant plusieurs jours avant utilisation pour permettre à la surface de devenir complètement sèche. Une boucle mesurant $1 \mathrm{~mm}$. de diamètre et faite de fil de platine de $0 \mathrm{~mm} .3$ de diamètre est employée

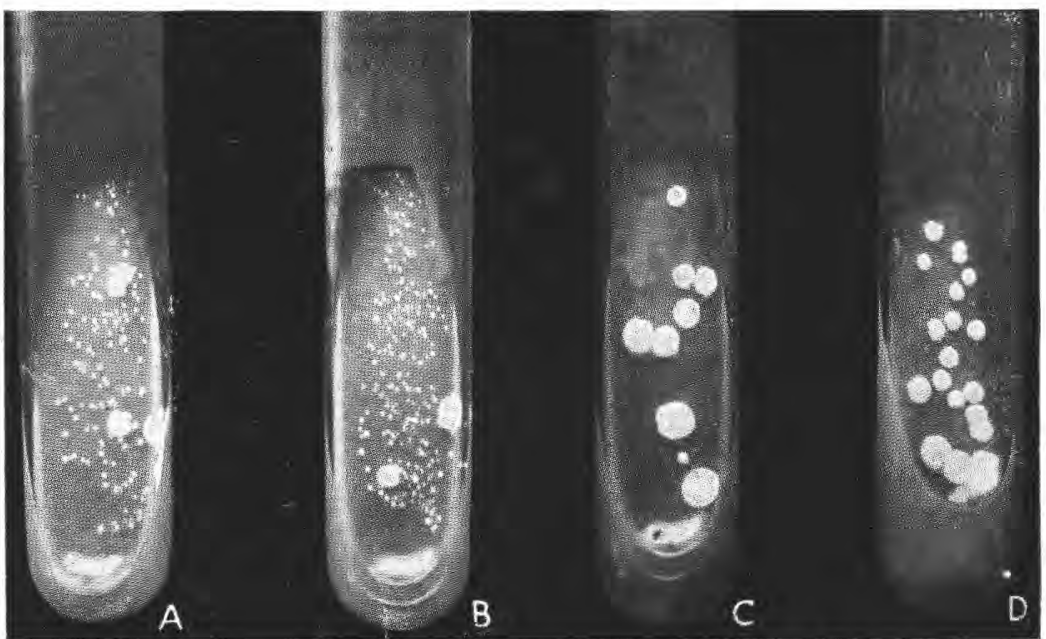

Fig. 2. Tubes inclinés de Burri préparés avec du lait frâ̂chement trait.

$\mathrm{A}$ et $\mathrm{B}$ montrent une prédominance de colonies de Streptocoques ; C et D ne montrent que des colonies de Microcoques. Dans ce dernier cas, les colonies peuvent être, soit blanches, soit jaunes, soit oranges, suivant le type de microcoque présent.

(1) Proc. World's Dairy Congress, London, 690-696, 1928.

(2) $500 \mathrm{gr}$. de veau maigre finement hachés sont mis à macérer pendant la nuit dans 1 litre d'eau : la macération est chauffée à 20 livres de pression $11 \mathrm{~kg} .4$ ou $127^{\circ} \mathrm{C}$ ) pendant 20 minutes, filtrée, additionnée de $5 \mathrm{gr}$. de chlorure de sodium et 5 gr. de peptone Bacto, la réaction amenée au $p H$ 7,2; le bouillon est chauffé à 20 livres de pression (1 kg. 4 ou $127^{\circ} \mathrm{C}$ ) pendant 15 minutes, filtré et stérilisé. 
pour ensemencer ces plans inclinés. On prend une boucle pleine de lait et on touche la surface du plan incliné en trois places, en commençant au bas du plan. La substance à ensemencer est ensuite répandue sur la surface entière. La boucle peut être étalonnée en comparant les numérations obtenues avec des numérations obtenues sur plaques-étalons.

Après incubation, les colonies bien nettes sur les plans inclinés peuvent être examinées et comptées. Cette méthode est une des meilleures méthodes de culture pour déceler les vaches ayant des streptocoques dans la mamelle. Si des streptocoques sont trouvés par cette méthode, on peut certainement en conclure qu'il y a infection.

Il s'agit là d'une méthode de laboratoire, utilisée au mieux par les techniciens ayant l'habitude du travail de laboratoire.

\section{MÉTHODES DES PLAQUES DE PETRI.}

La méthode ordinaire des plaques peut être employée pour déceler le lait infecté.

Méthode. - Le mode opératoire peut être similaire à celui décrit dans les Méthodes officielles d'analyse du lait par l'Association américaine d'Hygiène publique. L'agar spécialement préparé dans ce but, ainsi que le milieu au bouillon de veau indiqué ci-dessus, a été aussi trouvé très utile. Outre ce milieu, un grand nombre d'autres milieux ont été proposés, tels que le milieu au krystall violet par BRYAN (1) et l'agar alcali-albuminate-saccharose-sérum par KLIMMER, HAUPt et Roots (2). Si un milieu au sang doit être employé, $5 \%$ de sang de cheval récemment défibriné doivent être ajoutés après que l'agar a été refroidi à $40^{\circ}$ juste avant la mise en tubes.

La présence de colonies de streptocoques sur ces agars indique une infection si l'échantillon examiné ar été extrait directement d'une mamelle sans contamination possible par les streptocoques lactiques normaux.

Utilisation du test. - Les méthodes de culture pour déceler les infections de la mamelle ne sont pas aussi délicates que certaines autres méthodes. Pour cette raison, la présence de colonies de streptocoques sur les plaques d'agar en utilisant n'importe lequel des milieux mentionnés plus haut devrait indiquer qu'il y a infection (3).

(1) Amer, Jour. Public Health, 22, 749, 1932.

(2) Centralbl. f. Bakteriol., I Abt., Orig., 107, 206, 1928.

(3) Les méthodes décrites ici se réfèrent entièrement à la recherche de la mammite chronique et aiguë. Si le type de mammite à mal de gorge septique se présente, sa présence sera ordinairement nette, en raison d'une infection parmi les consommateurs de lait. Dans ce cas, les colonies de Streptocoque sont isolées, soit de tubes inclinés d'agar de Burri ou de plaques d'agar-sang. Si on utilise les premiers, les colonies sont repiquées en bouillon et éventuellement sur plaques agar-sang. Les streptocoques bêta-hémolytiques sont ensuite étudiés ultérieurement pour déterminer s'ils sont du type humain. 


\section{TEST DES CHLORURES.}

L'essai des chlorures est connu depuis quelque temps et est une des méthodes chimiques les plus élémentaires. Elle a été décrite par Hammer et BAILey (1) pour être utilisée dans le lait anormal. L'essai est basé sur le fait que les tissus de la mamelle infectés permettent aux sels du plasma du sang de s'infiltrer dans le lait fraîchement sécrété. Une augmentation au-dessus de la normale de la proportion de chlorure de sodium dans le lait frâichement trait indique par conséquent un état anormal de la mamelle.

Mode opératoire - $10 \mathrm{~cm}^{3}$ de lait dans un flacon d'Erlenmeyer sont dilués avec $40 \mathrm{~cm}^{3}$ d'eau distillée. 8 à 10 gouttes d'une solution à $10 \%$ de chromate de potassium sont ajoutées. Le lait dilué est titré au point final désiré avec du nitrate d'argent N/10. La proportion de chlorure présent est déterminée en considérant que $1 \mathrm{~cm}^{3}$ de nitrate d'argent employé correspond à $0 \mathrm{mmgr} .355$ de chlore.

Une méthode plus rapide a été présentée par HAYDEN (2). Elle peut être utilisée à la ferme. Mesurer exactement $5 \mathrm{~cm}^{3}$ d'une solution de nitrate d'argent ( 1 gr. 3415 de nitrate d'argent dans 1 litre d'eau) dans une petite bouteille ou un tube. Ajouter 2 gouttes d'une solution de chromate de potassium à $10 \%$, puis ajouter exactement $1 \mathrm{~cm}^{3}$ de lait. Deux autres méthodes abrégées sont aussi suggérées par HAYDEN.

Dans la méthode de titrage, le point final de la réaction est déterminé par le changement de coloration du lait, d'orange foncé au jaune citron. Dans les conditions générales, on admet que tout lait montrant plus de $0,14 \%$ de chlorures a été obtenu de mamelles infectées. Dans l'essai abrégé, les réactions sont arrangées de telle façon qu'une couleur jaune apparaît dans le mélange si la concentration des chlorures est plus élevée que cette proportion.

La véritable interprétation des résultats de cet essai est quelque peu difficile. Les experts ne sont pas d'accord sur la proportion normale du lait en chlorures. Cependant, il est admis généralement que la teneur normale du lait en chlorures varie de 0,09 à $0,14 \%$. Certains observateurs ont décidé de considérer que tout lait contenant une proportion de chlorures plus élevée que $0,16 \%$ provient de mamelles infectées.

Utilisation du test. - Ce test est probablement un des plus précis en usage pour déceler des mamelles infectées s'il est exécuté convenablement. En réalité, il est si délicat que beaucoup d'infections minimes de la mamelle peuvent donner des résultats positifs.

(1) Iowa State College of Agr. and Mechanic Arts, Bul. n० 41, 1917.

(2) Cornell Veterinarian, 22, 277, 1932. 
Dans l'exécution de ces essais, il y a certaines difficultés. Le point final de la réaction est difficile à déterminer. Un expérimentateur de laboratoire ne peut obtenir des résultats comparatifs qu'avec beaucoup de pratique, car ce point final n'est discerné qu'avec habileté et expérience.

\section{TEST DE LA CATALASE.}

L'épreuve de la catalase fut imaginée par TrommsdorfF (1) pour déceler le lait provenant de mamelles anormales. L'épreuve est basée sur le fait que le tissu cellulaire contient une certaine proportion de l'enzyme, connue sous le nom de catalase, qui réduit l'eau oxygénée en eau et oxygène. Pratiquement, dans tout lait provenant de mamelles infectées, il y a un grand nombre de globules blancs. L'épreuve devient done un indicateur grossier du nombre de globules qui existent dans le lait. Cependant, dans certains cas isolés, une épreuve de la catalase positive peut être obtenue alors qu'on ne trouve qu'un petit nombre de leucocytes.

Mode opératoire. - A $15 \mathrm{~cm}^{3}$ de lait dans un tube fermentateur de Smith sont ajoutés $5 \mathrm{~cm}^{3}$ d'une solution d'eau oxygénée à $1 \%$ et le mélange, laissé pendant 3 heures à $37^{\circ} \mathrm{C}$. La quantité de gaz dans le bras fermé est notée. Plus de 1,5\% de gaz dans le tube coudé indique que le lait a été obtenu d'une mamelle anormale.

Le type de tube employé pour cette épreuve varie : on utilise par exemple le tube fermentateur de Smith, le tube fermentateur de Durham, et plusieurs types spéciaux différents.

Une épreuve plus rapide a été proposée comme suit : placer une grosse goutte de lait sur une plaque noire. Ajouter une goutte d'une solution à $10 \%$ d'eau oxygénée. Des bulles apparaissent dans la goutte dans le cas de lait provenant de mamelles infectées.

En se plaçant au point de vue pratique, il n'est probablement pas possible d'utiliser les résultats positifs obtenus dans cette épreuve pour éliminer les individus infectés du troupeau. L'épreuve, telle qu'elle est employée actuellement, est trop délicate, et trop de vaches seraient condamnées qui n'ont pas une infection suffisante pour exiger leur isolement et leur élimination. Si un programme de contrôle de la mammite arrive à un stade tel que tous les individus portant une infection doivent être éliminés, il faudra que cette épreuve soit d'une valeur très nette

\section{TEST DU LACTOSE.}

Beaucoup de chercheurs ont utilisé un test déterminant la proportion de lactose dans le lait pour déceler des conditions anormales. Roselu (2) a noté son application possible dans le con-

(1) Berliner Tierarztl, Wochen,, 22, 282, 1906.

(2) Cornell Veterinarian, 21, 80, 1931. 
trôle de la mammite. L'épreuve est basée sur le fait connu que la quantité de lactose dans le lait provenant de mamelles malades est inférieure à celle du lait provenant de mamelles normales.

Méthode. - A $2 \mathrm{~cm}^{3}$ du lait à essayer on ajoute $1 \mathrm{~cm}^{3}$ d'une solution de soude à $25 \%$. On amène le mélange à l'ébullition et on continue jusqu'à ce qu'une couleur foncée soit obtenue. La proportion de lactose est déterminée par comparaison avec un étalon de couleur préparé en traitant divers échantillons de lait contenant des proportions connues de lactose de la même façon. Cet essai est un essai grossier pour la détermination du lactose et il n'est pas précis au delà d'une variation de plus de $0,5 \%$.

Une proportion de moins de $4 \%$ de lactose est considérée comme indiquant que le lait a été obtenu d'une mamelle anormale.

Utilisation du test. - L'épreuve n'est utilisable qu'au laboratoire et, dans de tels cas, n'est pas considérée comme étant une méthode satisfaisante pour déceler le lait de quartiers infectés. Elle exige du temps et n'est pas suffisamment précise pour les travaux de recherches.

\section{NUMÉRATION DES LEUCOCYTES.}

Les modifications pathologiques de la mamelle sont généralement mises en évidence par le passage dans le lait des globules blancs (leucocytes) du sang. Le nombre de leucocytes trouvé indique généralement la gravité de l'infection.

Méthode. - L'examen microscopique direct imaginé par Prescott et Breed (1) peut être utilisé pour déceler les leucocytes dans le lait au moment où il est extrait du pis. La méthode est donnée dans les Méthodes officielles pour l'analyse du lait par l'Association américaine d'Hygiène publique. Les leucocytes sont comptés et leur nombre noté.

On a trouvé que tout lait ayant plus de 120.000 leucocytes par centimètre cube provient d'une mamelle ayant une modification pathologique du tissu. D'un autre côté, il n'est pas correct de considérer tout lait ayant un taux cellulaire supérieur à ce nombre comme anormal. Le lait ayant un nombre de cellules supérieur à 500.000 par centimètre cube provient presque toujours d'une mamelle infectée.

Utilisation du test. - Le test doit être réservé au laboratoire et doit être effectué par un expérimentateur habitué. C'est un des essais les plus délicats et les plus précis pour déceler du lait anormal.

(1) Jour. Inf. Dis., 7, 632, 1910. 


\section{EXAMEN MICROSCOPIQUE DIRECT.}

L'examen microscopique direct du lait fut proposé par BREED (1) et est généralement adopté pour les examens réguliers du lait commercial. Son utilisation pour déceler la mammite au laboratoire a été fortement recommandée, et elle a rendu d'excellents services dans certains travaux de laboratoire.

Méthode. - Les grandes lignes de la technique peuvent être trouvées dans les Méthodes officielles d'analyse du lait de l'Association américaine d'Hygiène publique (2).

La présence de streptocoques en longues chaînes et de leucocytes dans les préparations faites avec du lait fraîchement trait indique qu'il y a une infection active.

Utilisation du test. - On a trouvé que cette méthode décèle rapidement les vaches souffrant d'une infection avancée de la mamelle. Quand on l'emploie sur du lait frais, on a aussi trouvé que si l'infection est suffisamment grave pour montrer des streptocoques en longues chaînes, l'examen attentif direct du lait du quartier infecté montre que l'état est ordinairement suffisamment grave pour que le lait présente des grumeaux. Ce n'est que rarement qu'on trouvera par cette méthode des streptocoques du lait de quartiers individuels ayant une apparence normale. Du lait mélangé des quatre quartiers peut être normal en apparence et montrer des streptocoques en longues chaînes à l'examen microscopique direct. La méthode trie Jes cas graves.

Cet essai a des utilisations très précieuses pour déceler et confirmer la mammite. Dans les endroits où on n'a pas encore institué de contrôle de la mammite, cette méthode peut être employée au laboratoire pour déceler rapidement les cas les plus prononcés. C'est une des méthodes les plus usitées dans de telles conditions, et elle donne d'excellents résultats. Elle est habituellement employée en même temps que le contrôle de laboratoire régulier des fournitures de lait. Si des streptocoques en longues chaînes sont trouvés dans le lait de mélange, il est habituellement possible de retrouver l'origine de ces organismes et d'éliminer la vache infectée.

L'essai est essentiellement un essai de laboratoire et a ainsi sa plus grande utilisation, quoique du matériel transportable pour être utilisé à la ferme existe à l'usage des personnes qui ont l'habitude de ces travaux.

\section{MISE A L'ÉTUVE DES ÉCHANTILLONS.}

On sait qu'une vache peut être infectée et qu'il peut n'exister

(1) Centralbl.f. Bakteriol., II Abt., 30, 337-348, 1911.

(2) New York American Public Health Association, 5e éd., 1929. 
pourtant qu'un très petit nombre de streptocoques, de sorte que ceux-ci peuvent passer inaperçus lors de l'examen direct des échantillons fraîchement traits ainsi que lors des diverses méthodes de culture habituellement employées. Pour cette raison, l'examen d'un échantillon mis à l'étuve peut être employé pour déceler les cas obscurs.

Méthode. - On se procure des échantillons fraîchement traits d'une façon aseptique de chaque quartier et on les laisse à l'étuve, pendant la nuit, à $37^{\circ} \mathrm{C}$. Les préparations sont examinées microscopiquement, en ce qui concerne la présence de streptocoques en longues chaînes, après cette période d'incubation.

Dans l'examen de ces échantillons, il faut bien s'attacher à différencier convenablement les streptocoques en courtes chaînes et les streptocoques en longues chaînes. Beaucoup de streptocoques en courtes chaînes qui sont la cause de l'acidification normale du lait peuvent se développer. Ces organismes n'ont pas de rapport avec les infections de la mamelle et doivent être négligés. Les streptocoques en longues chaînes doivent être considérés comme ayant de l'importance, surtout ceux ayant des chaînes de 10 unités ou plus. Aucune importance ne doit être accordée à l'absence de leucocytes dans cet examen, car, pendant la période d'incubation, les leucoeytes peuvent être dissous par les enzymes normalement présentes.

Utilisation du test. - Toutes les préparations faites par cette méthode et présentant des streptocoques en longues chaînes doivent être considérées comme provenant d'individus infectés. Si on ne trouve pas de streptocoques en longues chaînes dans les échantillons fraîchement traits et si on n'en note pas dans les échantillons incubés (1), l'infection est ordinairement d'un type inactif, mais on doit considérer la vache comme ayant une infection sous-clinique.

\section{BOL D'ESSAI DE TRAITE.}

Le bol d'essai de traite (fig. 3) fut indiqué pour la première fois par MoAk (2) comme susceptible de déceler les quartiers infectés des vaches dans les fermes produisant du lait certifié. Il a été adopté sur une grande échelle dans certaines régions, et peut être utilisé comme étant un essai assez précis pour déceler des quartiers ayant des infections avancées ou graves.

Méthode. - Le bol d'essai de traite (3) est un récipient sur

(1) Cette méthode a été de plus en plus utilisée dans les laboratoires de la Station depuis 1911 et fut mentionnée pour la première fois dans le Bulletin technique no 80 par BAKEI et BREED (1920).

(2) Cornell Veterinarian, 6, 36-40, 1912.

(3) Des bols d'essai de traite peuvent être obtenus de The Irisk Manujacturing Company, Canandaigua, N. Y., U. S. A. 
lequel un tamis de fil métallique de maille 100 à 120 a été placé. 3 ou 4 jets du premier lait sont traits à travers le tamis. La présence de quartiers fortement infectés est décelée par l'apparition sur le tamis de "flocons" ou petites masses coagulées. Dans les cas les plus graves, le lait passe à travers le tamis avec difficulté.

La présence des flocons doit être interprétée comme indiquant qu'il y a infection prononcée. On a prouvé que tous les quartiers donnant une réaction positive étaient infectés. Toutefois, tous les quartiers infectés ne donnent pas une réaction positive.

Utilisation du test. - Si un propriétaire désire suivre attentivement son troupeau, il doit utiliser le bol journellement. Cet essai est particulièrement exact si on utilise une machine à traire. Les vaches donnant du lait montrant des flocons doivent être considérées eomme dangereuses pour le reste du troupeau au point de vue de la dissémination de la mammite et doivent être isolées ou placées en fin de traite, et traites après le reste du troupeau.

Ce test est vraisemblablement le plus pratique de ceux utilisés à la ferme par le laitier. Toutefois, il est l'un des moins sensibles des divers tests qui peuvent être utilisés.

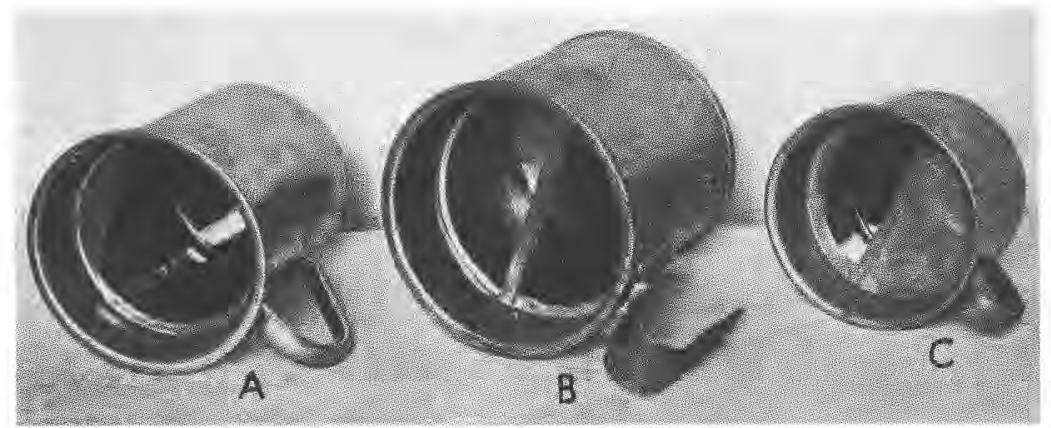

Fig. 3. Bol d'essai de traite.

$A$, modèle de la Dairymens League; $B$, modèle de Sheffield; $C$, modèle Borden

\section{RECHERCHE DU TISSU MAMMAIRE INDURE (FIBREUX, CICATRICIEL) ET EXAMEN PHYSIQUE POUR DECELER LA MAMMITE.}

Des vétérinaires intéressés au diagnostic des infections de la mamelle ont amélioré la méthode proposée par UDALL et JoHrson (1) pour trouver le tissu induré ou cicatriciel dans la mamelle par palpation manuelle.

(1) Comell Veterinarian, 21, 190, 1931. 
Méthode. - L'examen doit avoir lieu immédiatement après la traite, car un examen n'est pas satisfaisant quand la mamelle est distendue par le lait ou quand elle est congestiunféé et gonflée avant et après raffermissement. UDALL et JoHNSON décrivent cette méthode comme suit :

"Observez la symétrie de la mamelle. Levez chaque quartier avec les deux mains, en observant la dimension, le poids et le consistance, et en les comparant avec ceux de chacun des autres quartiers. Quand on trouve une différence dans la dimension des quartiers, comme par exemple un quartier de face grand et mou et un aussi de face petit et dur, le petit peut avoir été le siège d'une mammite aiguë qui a causé l'arrêt de la sécrétion du lait et la contraction du tissu glandulaire, mais le degré de modification inflammatoire peut n'être déterminé que par son activité lors des conceptions ultérieures. Si cependant on trouve que le plus petit quartier est nettement nodulaire, au lieu d'être lisse et régulier à la surface, on peut conclure que du tissu conjonctif a remplacé le tissu glandulaire ; il s'est induré. Pendant le stade actif d'une inflammation, un quartier atteint peut être bien plus grand que son voisin; si un quartier postérieur est nettement plus grand que l'autre, un doute peut exister en ce qui concerne la question de savoir si l'un est enflé et induré à un degré pathologique ou si l'autre est atrophié pour la même raison. En règle générale, la question est facilement réglée par la présence d'indurations irrégulières. Mais si, éventuellement, il y a doute, une décision peut être prise à l'aide de l'essai colorimétrique. Il est bien évident qu'une longue pratique dans la palpation des mamelles donne une dextérité accrue.

"Ensuite, appliquez la palpation superficielle à la surface du quartier; chaque quartier doit être normalement lisse et lobulé. Une surface normalement lobulée est de contour uniforme et les saillies sont petites et régulières, tandis qu'une lobulation pathologique n'est pas distribuée régulièrement et que les lobules n'en sont pas de dimension uniforme.

"La palpation profonde est pratiquée en soulevant à nouveau chaque quartier et en pétrissant vigoureusement en profondeur toutes les parties pour déceler les indurations limitées ou diffuses et reconnaître l'élasticité et la souplesse du tissu glandulaire normal. "

Utilisation du test. - Il est difficile d'interpréter convenablement les résultats obtenus par un tel examen manuel de la mamelle. La signification de ce tissu cicatriciel ou induré n'est pas nettement connue, quoi qu'on puisse conclure avec certitude qu'un fort pourcentage des animaux qui ont de l'induration sont infectés et doivent être éliminés du troupeau. 
Cette méthode ne peut être utilisée que par des vétérinaires qualifiés pour de tels examens. Même des vétérinaires pratiquant normalement leur art ont besoin d'une expérience spéciale pour effectuer ces examens d'une manière satisfaisante.

Les vétérinaires combinent fréquemment les examens de la mamelle avec l'essai au bleu de bromothymol et le bol d'essai de traite. Cet examen consiste d'abord en l'épreuve du bol d'essai de traite suivi par le test au bleu de bromothymol; puis la mamelle est vidée et une palpation manuelle est effectuée. Les trois essais sont ensuite comparés et, en conclusion, on peut, d'après Udall et Johrson (1), les classer en quatre groupes comme suit:

Groupe 1: Vaches exemptes d'induration et ne donnant pas de réactions positives avec les autres essais. Ce groupe est appelé groupe normal.

Groupe 2 : Ici se place le groupe suspect. Le lait des vaches de ce groupe peut donner une réaction positive au bol d'essai de traite et au bleu de bromothymol. Un examen ultérieur du lait au laboratoire peut montrer des streptocoques. Le lait peut être d'apparence normale. Il peut y avoir là une antérieure "maladie du pis" (en anglais : garget). La palpation peut montrer une ou plusieurs zones dures limitées ou des lésions. Les quartiers peuvent manquer d'uniformité en dimension et en consistance. L'animal peut devenir normal ou passer au groupe trois.

Groupe 3 : La mamelle est nettement et uniformément anormale à la palpation et tous les autres essais peuvent donner des réactions positives. Le lait peut être encore d'apparence normale. Les vaches de ce groupe ont habituellement une antériorité d'attaques répétées de "maladies du pis".

Groupe 4: Ici nous avons le groupe de l'infection nette. La mamelle est tellement malade que l'infection est décelable par tout laitier ou propriétaire tant soit peu intelligent. Le lait présente de nombreux grumeaux, et peut paraître ou aqueux ou épais. On trouve généralement des streptocoques à l'examen au laboratoire. La production peut être diminuée.

Ces groupements doivent être effectués par un vétérinaire compétent et si ses classifications doivent être exactement précisées, il devra se faire aider par un laboratoire. Ces classifications ne peuvent pas être effectuées d'une façon convenable par un laitier quelconque.

\section{TEST DE TROMMSDORFF.}

La méthode exposée par Trommsdorff (2) est basée sur ce prin-

(1) Loc. cit.

(2) Münchener Med. Wochen., 53, 1, 540, 1906. 
cipe que les cellules et la boue du lait peuvent être centrifugées, leur proportion mesurée dans le tube spécial utilisé, et leur qualité déterminée par l'examen microscopique du culot de centrifugation.

Méthode. - $10 \mathrm{~cm}^{3}$ de lait sont introduits dans un tube de verre spécial dont l'extrémité est étirée en un tube capillaire gradué en millièmes de centimètre cube. Le tube est placé dans une tête spéciale de centrifuge qu'on fait tourner à 1.200 tours par minute.

On lit le résultat en notant la quantité de boue déposée. Le lait normal donne à peu près de $0 \mathrm{~cm}^{3} 002$ à $0 \mathrm{~cm}^{3} 004$, tandis quelelait provenant de mamelles malades fournira $0 \mathrm{~cm}^{3} 01$ ou plus. La proportion de dépôt est importante, mais non concluante. Ultérieurement à la publication de la méthode originale, on a trouvé qu'elle serait plus précise si un examen microscopique du dépôt était effectué pour déterminer Ja présence ou l'absence de cellules ou de streptocoques en longues chaînes. Toutefois, comme il a été énoncé à l'origine, le test est plutôt de nature quantitative que de nature qualitative.

Tous les cas dans lesquels des streptocoques en longues chaînes sont trouvés dans le dépôt doivent être considérés comme provenant de mamelles infectées. Toutefois, si le lait avant centrifugation ne contient pas un nombre excessif de leucocytes ou de streptocoques, leur présence dans le dépôt centrifugé indique que l'infection n'est pas active. Quand on l'utilise comme méthode quantitative, l'essai est inexact, car un très grand nombre de cellules peuvent monter avec la crème pendant la centrifugation. Quoique la méthode soit un procédé utile de mesure de la proportion de boue, personne n'a prouvé qu'il existait un rapport entre la proportion de boue dans les échantillons centrifugés et le nombre de cellules dans le lait original.

Utilisation du test. - Le test est un de ceux qui ne sont utiles qu'au laboratoire. Il peut être utile comme essai qualitatif dans les cas où une recherche spéciale a besoin d'être effectuée relativement à un groupe particulier d'animaux.

\section{TEST STEWART-SLACK.}

La présence d'un nombre excessif de leucocytes et de streptocoques (1) dans le lait peut être déterminée par la méthode du dépôt gras de Stewart-Slack.

Méthode. - La technique peut être trouvée dans les Méthodes officielles d'analyse du lait établies par l'Association américaine d'Hygiène publique (5e édition, 1927). Le nombre de leucocytes et de streptocoques en longues chaînes est noté et leur présence interprétée comme signifiant la présence d'une infection de la mamelle.

(1) Voir le Bulletin no 380, New York Agr. Exp. Stat., qui discute le développement historique de ce test. 
Utilisation du test. - Moyen commode d'examiner les dépôts centrifugés.

\section{RÉSULTATS OBTENUS.}

\section{ORIGINE DES PRODUITS ÉTUDIES.}

Afin d'étudier l'incidenee de la mammite ehronique dans les districts laitiers aussi bien que la valeur des méthodes de recherche, 221 vaches condamnées par l'épreuve de la tubereuline furent choisies pour étude. De 1 à 4 échantillons de lait furent obtenus de chaque quartier de ces animaux. Les échantillons furent refroidis et apportés au laboratoire pour les déterminations habituelles généralement employées pour déceler la mammite chronique, c'est-à-dire : numération des cellules, des colonies apparaissant sur le bouillon de veauagar avec et sans sang, des colonies apparaissant sur les plaques d'agar de Burri, examen microscopique direct, examen microscopique après mise à l'étuve pendant la nuit à $37^{\circ} \mathrm{C}$., dosage des chlorures, du lactose et de la catalase présents, réaction au bleu de bromothymol. Les examens furent habituellement effectués moins de 4 heures après que les échantillons avaient été traits.

Les échantillons de chaque quartier furent obtenus après que cinq ou six jets du premier lait avaient été rejetés.

$\mathrm{Au}$ moment où les échantillons étaient prélevés, on utilisait aussi un bol d'essai de traite, et un examen physique de la mamelle était effeetué.

Pour augmenter encore l'étude de la valeur des tests de la mammite, des échantillons de quartiers furent prélevés sur environ 170 vaches pendant une période de près de deux ans. De nombreux essais furent effectués sur ces échantillons et les résultats confrontés avec un examen vétérinaire physique ordinaire et avec l'histoire clinique de chacune des vaches qui furent soigneusement examinées et notées journellement.

\section{EXAMEN POST-MORTEM.}

Au moment de l'abattage, les mamelles furent complètement enlevées de chaque animal. Ensuite, chaque quartier fut soigneusement disséqué et on nota la présence ou l'absence de tissu fibreux ou d'induration. On fit aussi attention à noter s'il y avait du pus dans les canaux galactophores ou dans les sinus.

Dans un grand nombre de cas, des tissus furent prélevés pour être étudiés histologiquement par fixation dans le liquide de Zenker. Quand ce fut possible, des eultures furent préparées avec le pus pour servir à des études ultérieures 
NOMBRE DE VACHES INDIQUE PAR LES MÉTHODES DE CULTURE COMME PRODUISANT DU LAIT CONTENANT DES STREPTOCOQUES. Dans un examen de 439 quartiers qui ne présentaient pas de preuve clinique de mammite, $46 \%$ furent trouvés (tableau 1) fournissant des streptocoques, comme le montrèrent les développements des colonies semblables à des streptocoques sur bouillon de veau et sur bouillon de veau-sang de cheval-agar. En classant ces mamelles comme ne montrant pas de preuve clinique de la mammite, il fut déterminé qu'il n'y avait pas inflammation active évidente des quartiers. La mamelle était d'apparence externe normale. Toutefois, la plupart de ces quartiers montrèrent des degrés variés de sclérose et d'induration sans preuve de mammite aiguë caractérisée.

TABLEAU 1.

NOMBRE DE QUARTIERS DE VACHES, SANS PREUVE ÉVIDENTE DE MAMMITE, DONNANT DES STREPTOGOQUES DANS LEUR LAIT.

\begin{tabular}{l|c|c}
\hline \hline & $\begin{array}{c}\text { Nombre } \\
\text { de quartiers }\end{array}$ & $\begin{array}{c}\text { Pourcentage } \\
\text { de quartiers }\end{array}$ \\
\cline { 3 - 4 } Streptocoques absents .... & 239 & 54 \\
Streptocoques présents $\ldots . .$. & 200 & 46 \\
\hline \hline
\end{tabular}

TABLEAU 2.

DISTRIBUTION DES STREPTOCOQUES ET DU PUS CHEZ LES VACHES.

\begin{tabular}{|c|c|c|c|}
\hline 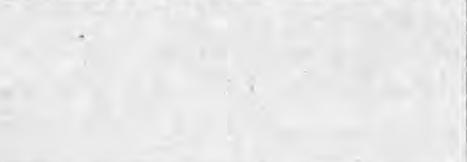 & $\begin{array}{l}\text { Nombre } \\
\text { devaches }\end{array}$ & $\begin{array}{l}\text { Pourcentage } \\
\text { de vaches }\end{array}$ & $\begin{array}{l}\text { Pourcentage } \\
\text { de vaches } \\
\text { montrant du } \\
\text { pus à l'autop- } \\
\text { sie }(\mathrm{I})\end{array}$ \\
\hline $\begin{array}{c}\text { Aucun streptocoque dans aucun } \\
\text { quartier } \ldots \ldots \ldots \ldots \ldots \ldots \ldots\end{array}$ & 26 & 22 & 3 \\
\hline $\begin{array}{l}\text { Des streptocoques dans un ou } \\
\text { plusieurs quartiers } \ldots \ldots \ldots \ldots\end{array}$ & 92 & 78 & 18 \\
\hline $\begin{array}{l}\text { Des streptocoques dans un quar- } \\
\text { tier } \ldots \ldots \ldots \ldots \ldots \ldots \ldots \ldots \ldots \\
\text { Des streptocoques dans deux }\end{array}$ & 40 & 34 & 17 \\
\hline $\begin{array}{l}\text { quartiers } \ldots \ldots \ldots \ldots \ldots \ldots \ldots \\
\text { Des streptocoques dans trois }\end{array}$ & 35 & 30 & 25 \\
\hline $\begin{array}{c}\text { quartiers } \ldots \ldots \ldots \ldots \ldots \ldots \ldots \\
\text { Des streptocoques dans quatre }\end{array}$ & 13 & 11 & 33 \\
\hline quartiers $\ldots \ldots \ldots \ldots \ldots \ldots$ & 4 & 3 & 25 \\
\hline
\end{tabular}

(1) $21 \%$ de toutes les vaches montrèrent du pus dans un ou plusieurs quartiers. 
Les plaques d'agar indiquaient que les streptocoques variaient, en nombre, de quelques unités à 100.000 ou plus par centimètre cube. La plupart des quartiers donnant des streptocoques en contenaient moins de 100.000 par centimètre cube de lait. $12 \%$ seulement du nombre total des quartiers donnèrent des streptocoques en nombre supérieur à 100.000 par centimètre eube. Une étude postmortem (tableau 2) des quartiers qui donnaient des streptocoques en n'importe quelle proportion dans le lait indiqua que $18 \%$ de ces quartiers contenaient du pus. D'un autre côté, seulement $3 \%$ des quartiers qui ne montrèrent pas de preuve évidente de streptocoques dans les cultures contenaient du pus à l'autopsie.

Il est probable que les $46 \%$ des quartiers qui donnaient des streptocoques, comme l'indiquait la culture sur plaques d'agar, étaient des sources potentielles d'infection pour des quartiers normaux. On peut aussi supposer que quand l'infection a atteint le stade de suppuration, elle a probablement été d'une assez longue durée et peut être difficile à éliminer.

PRÉSENOE dE PUS dANS LeS MAMELLES. - Un examen postmortem de 560 quartiers indiqua que dans $11 \%$ de ces quartiers (tableau 3) il y avait présence de poches de pus, tandis que $21 \%$ des vaches autopsiées montrèrent du pus dans un ou plusieurs quartiers.

TABLEAU 3.

NOMBRE DE QUARTIERS DE VACHES SANS PREUVE CLINIQUE DE MAMMITE QUI MONTRÉRENT DU PUS A L'AUTOPSIE.

\begin{tabular}{|c|c|c|}
\hline \multirow{2}{*}{$\begin{array}{c}\text { Nombre de quartiers } \\
\text { examinés }\end{array}$} & \multicolumn{2}{|c|}{ Quartiers montrant du pus à l'autopsie $(I)$} \\
\hline & Nombre & $0 / 0$ \\
\hline $460 \ldots \ldots \ldots \ldots \ldots \ldots$ & 51 & 11 \\
\hline
\end{tabular}

On peut normalement se poser la question de savoir si la présence de poches de pus dans le tissu mammaire peut être décelée par une étude du lait. Un rapprochement des renseignements fournis par l'examen au laboratoire du lait obtenu de ces 51 quartiers indiqua qu'aucun des tests ordinaires du laboratoire ne pouvait déceler la présence de pus ou de poches de pus dans le tissu mammaire avee quelque degré de précision. Le lait de $32 \%$ seulement de ces quartiers donna quelque indication d'irrégularité quand on utilisa ou le bol d'essai de traite ou des plaques d'agar-sang ; $38 \%$ montrèrent la présence d'une proportion anormale de eatalase, tandis que $67 \%$

(1) Vaches montrant du pus (post-mortem) dans un ou plusieurs quartiers, $21 \%$. 
montrèrent une proportion de chlorures supérieure à la normale. Cependant, $81 \%$ de ces 51 quartiers montrèrent, outre la présence de pus à l'examen post-mortem, l'existence de tissus fibreux ou indurés, indiquant que le quartier subissait ou avait subi une infection générale à quelque époque antérieure.

Il ne semble pas qu'il existe actuellement de méthode de laboratoire d'examen du lait permettant de déceler exactement les quartiers recélant des poches de pus au sein de leurs tissus.

SIGNIFICATION DU NOMBRE DES CELLULES EXISTANT DANS LES INFECTIONS DE LA MAMELLE. - On a beaucoup discuté sur la signification des cellules dans le lait. On a supposé habituellement qu'un excédent dans le nombre des cellules indiquait une condition anormale.

Dans l'étude actuelle, un rapport entre le nombre des cellules trouvées dans le lait, et les autres tests utilisés pour déceler des conditions anormalés de la mamelle, indiqua qu'une signification très nette devait être attachée à la présence d'un nombre excessif de cellules. On a trouvé que si le chiffre de 1.000 .000 de cellules par centimètre eube était pris comme étalon arbitraire du maximum pouvant exister dans les échantillons de lait normal avant qu'un soupçon ne s'élève en ce qui concerne leur qualité bactériologique, aucune signification précise ne pouvait être donnée sur la numération des cellules pour déceler la mammite chronique. Quand, à la lumière des autres essais qu'il fut possible d'effectuer, on étudia tous les quartiers montrant une quantité de cellules supérieure à 1.000 .000 par centimètre cube, on ne trouva que peu de rapport entre la numération des cellules et les autres essais qui indiquent habituellement des cónditions anormales. D'un autre côté, si le chiffre de 3.000.000 de cellules par centimètre cube était fixé comme l'étalon arbitraire au-dessus duquel le lait pourrait être considéré comme douteux, on pourrait trouver un rapport très net entre la numération des cellules et certains autres tests employés pour déceler la mammite chronique.

$98 \%$ de tous les quartiers (tableau 4) qui contenaient plus de 3.000.000 de cellules par centimètre cube, avaient aussi une teneur en catalase accrue et renfermaient du tissu fibreux. Plus de $90 \%$ de ces quartiers montraient aussi une teneur en chlorures supérieure à la normale, une diminution de la proportion du lactose, et recélaient des streptocoques en longues chaînes dans les échantillons, mis à l'étuve pendant 10 heures ou plus longtemps.

Ces résultats indiqueraient qu'un nombre de plus de 3.000 .000 de cellules par centimètre cube correspond à une condition anormale de la mamelle, lorsque le nombre de cellules est examiné en comparaison 
TABLEAU 4.

SIGNIFICATION DU NOMBRE DE GELLULES EXISTANT DANS LES INFEGTIONS DES QUARTIERS.

\begin{tabular}{|c|c|c|c|c|c|c|c|c|c|}
\hline & & & POURCENT & AGE DONN & VANT DES I & RÉACTIONS & POSITIVES & & \\
\hline $\begin{array}{l}\text { NOMBRE DE CELLU- } \\
\text { LES EXISTANT PAR } \\
\text { CENTIMÈTRE CUBE }\end{array}$ & $\begin{array}{l}\text { Bleu de } \\
\text { bromo- } \\
\text { thymol }\end{array}$ & $\begin{array}{l}\text { Bol d'es- } \\
\text { sai de } \\
\text { traite }\end{array}$ & Catalase & $\begin{array}{l}\text { Moins de } \\
4 \% \text { de } \\
\text { lactose }\end{array}$ & $\begin{array}{l}\text { Plus de } \\
0,14 \% \text { de } \\
\text { chlorures }\end{array}$ & $\begin{array}{l}\text { Strepto- } \\
\text { coques } \\
\text { sur veau } \\
\text { agar }\end{array}$ & $\begin{array}{c}\text { Colonies } \\
\text { alpha ou } \\
\text { beta sur } \\
\text { agar- } \\
\text { sang }\end{array}$ & $\begin{array}{c}\text { Strepto- } \\
\text { coques en } \\
\text { longues } \\
\text { chaînes } \\
\text { dans } \\
\text { échantil- } \\
\text { lons mis } \\
\text { à l'étuve }\end{array}$ & $\begin{array}{c}\text { Sclérose } \\
\text { nette } \\
\text { post- } \\
\text { mortem }\end{array}$ \\
\hline Moins de $500.000 \ldots \ldots$ & 0 & 7 & 55 & 14 & 49 & 7 & 7 & 15 & 60 \\
\hline De 500.000 a 1.000 .000 . & 0 & 0 & 56 & 14 & 50 & 11 & 11 & 14 & 56 \\
\hline $\begin{array}{l}\text { De } 1.000 .000 \text { à } \\
2.000 .000 \ldots\end{array}$ & 10 & 35 & 80 & 50 & 60 & 15 & 30 & 45 & 80 \\
\hline Plus de $2.000 .000 \ldots$. & 25 & 60 & 75 & 60 & 37 & 37 & 27 & 27 & 70 \\
\hline Plus de $3.000 .000 \ldots \ldots$ & 50 & 50 & 98 & 91 & 94 & 46 & 50 & 90 & 98 \\
\hline
\end{tabular}


TABLEAU 5 .

RAPPORT ENTRE LE NOMBRE DE GELLULES PAR GENTIMÈTRE GUBE DANS LE LAIT ET LA PR ÉSENGE DE TISSU FIBREUX DANS LA MAMELLE.

\begin{tabular}{|c|c|c|c|c|c|c|c|c|c|}
\hline \multirow{2}{*}{$\begin{array}{l}\text { Proportion de } \\
\text { tissu fibreux }\end{array}$} & \multirow{2}{*}{$\begin{array}{c}\text { Nombre } \\
\text { de } \\
\text { quartiers } \\
\text { étudiés }\end{array}$} & \multirow{2}{*}{$\begin{array}{c}\text { Nombre } \\
\text { d'essais } \\
\text { sur } \\
\text { chaque } \\
\text { quartier }\end{array}$} & \multicolumn{7}{|c|}{ PoURCENTAGE D } \\
\hline & & & $\begin{array}{c}\text { Moins } \\
\text { de } \\
30.000\end{array}$ & $\begin{array}{c}30.000 \\
\text { à } \\
60.000\end{array}$ & $\begin{array}{c}60.000 \\
\text { à } \\
150.000\end{array}$ & $\begin{array}{c}150.000 \\
\text { à } \\
300.000\end{array}$ & $\begin{array}{c}300.000 \\
\text { à } \\
1.000 .000\end{array}$ & $\begin{array}{c}1.000 .000 \\
\text { à } \\
5.000 .000\end{array}$ & $\begin{array}{c}\text { Plus } \\
\text { de } \\
5.000 .000\end{array}$ \\
\hline Absence $\ldots \ldots \ldots \ldots$ & 63 & 20 & 87 & 12 & 1 & 0 & 0 & 0 & 0 \\
\hline Légère ..... & 68 & 20 & 60 & 3 & 7 & 9 & 7 & 9 & 5 \\
\hline Nette $\ldots \ldots \ldots . \ldots$. & 109 & 20 & 25 & 5 & 7 & 11 & 19 & 25 & 8 \\
\hline Marquée ..... & 24 & 20 & 22 & 5 & 0 & 0 & 18 & 34 & 21 \\
\hline
\end{tabular}

Nombre total d'essais : 5.080 . 
avec d'autres tests de laboratoire. Toutefois, un point plus important dans l'examen de la signification du nombre des cellules est la condition pathologique de la mamelle dont le lait provient. Afin d'obtenir des renseignements sur ce point, des échantillons hebdomadaires de quartiers furent obtenus de 150 vaches pendant une période de près de deux ans. Un examen physique détermina la présence ou l'absence de tissu fibreux. Le nombre des leucocytes dans le lait fut ensuite considéré dans ses rapports avec le tissu fibreux de la mamelle.

On a trouvé que pendant une période de 10 mois, aucun quartier exempt d'indurations ou de tissu fibreux ne donnait à un moment quelconque (tableau 5) plus de 150.000 cellules par centimètre cube. D'un autre côté, ce nombre ne fut, du reste, atteint que dans un seul cas isolé. $99 \%$ des quartiers normaux, c'est-à-dire des quartiers exempts de tissu cicatriciel, avaient moins de 60.000 cellules par centimètre cube, tandis qu'une grande majorité ( $87 \%$ ) ne donnèrent jamais un taux supérieur à 30.000 cellules par centimètre cube.

Par ailleurs, $63 \%$ des quartiers ayant du tissu fibreux donnèrent du lait contenant plus de 150.000 cellules par centimètre cube. Il est aussi intéressant de noter que $73 \%$ des quartiers ayant du tissu fibreux important donnaient plus de 500.000 eellules dans le lait.

On peut conclure avec certitude de ces résultats que tous les quartiers ayant par centimètre cube un nombre de cellules supérieur à 150.000 doivent être considérés comme suspects, s'il n'est pas définitivement prouvé qu'ils sont infectés de streptocoques.

On a aussi trouvé que tous les quartiers qui ont du tissu fibreux blane ne donnent pas tous un nombre appréciable de cellules. Environ $25 \%$ des quartiers classés comme ayant du tissu fibreux, net ou marqué, ne montrèrent dans aucun cas, au cours d'une série d'examens s'étendant sur dix mois, un nombre de cellules supérieur à 30.000 par centimètre cube. Il paraît évident qu'il y a deux groupes distincts de quartiers fibreux, ceux qui ne déchargent jamais de cellules, et ceux qui, constamment, pendant une longue période, donneront du lait dans lequel le nombre des cellules sera relativement élevé. A présent, on peut conclure avec certitude queles numérations de cellules supérieures à 150.000 par centimètre cube proviennent toujours de quartiers qui ont tant soit peu de tissu fibreux. Toutefois, tous les quartiers indurés ne donnent pas de fortes quantités de cellules.

On a observé en outre que dès qu'un quartier commence à décharger un grand nombre de cellules dans le lait, le nombre des leucocytes reste relativement élevé pendant des mois et même plus longtemps. Pendant une période de dix mois d'observations sur des 
quartiers à " taux cellulaires élevés ", le nombre des leucocytes n'est jamais descendu au-dessous de 1.000 .000 par centimètre cube. En d'autres termes, dès qu'un grand nombre de leucocytes est apparu dans le lait d'un quartier quelconque, ce nombre diminue rarement.

Les mêmes observations furent trouvées exactes en ce qui concerne le lait contenant un petit nombre de leucocytes. Le nombre maximum trouvé dans le lait provenant de quartiers ne présentant pas de tissu cicatriciel et étudiés pendant une période de dix mois, n'excéda jamais 200.000 par centimètre cube, les animaux restant, pendant la période d'essai entière, des vaches à " taux cellulaire bas 》.

On nota une certaine proportion de variation entre la teneur en leucocytes du lait des quatre quartiers de la même bête. Une des observations les plus intéressantes faite à cet égard fut qu'on trouva une plus grande variation dans le lait provenant de quartiers ayant un nombre excessif de leucocytes que dans le lait en contenant un nombre minimum.

(A suivre.)

\title{
ÉTUdE DE L'APPRÉCIATION DE LA QUALITÉ DES CASÉINES LACTIQUES
}

\author{
par \\ JEAN PIEN \\ Ingénieur chimiste I. C. R. \\ Docteur ès sciences \\ Directeur des laboratoires \\ des a Fermiers Réunis "

\section{MARO BERGIER} \\ Ingénieur (I. A. N.) \\ du laboratoire des caséines \\ des " Fermiers Réunis "
}

(Suite.)

Nous croyons avoir montré dans notre précédent article pourquoi l'analyse chimique ordinaire des caséines lactiques doit être considérée comme insuffisante à déceler certains défauts de fabrication. Nous avons proposé de faire appel à une méthode d'examen complémentaire, dont nous devons rappeler, en quelques mots, le principe.

L'examen systématique de chaque point de la fabrication de la caséine lactique conduit, selon nous, à admettre comme bases de travail les trois conditions suivantes:

$1^{\circ} \mathrm{Ne}$ produire aucune protéine dégradée à partir de la caséine initiale.

$2^{\circ}$ Ne fixer sur la molécule protéique aucun élément qui n'y soit lié naturellement.

$3^{\circ}$ Ne laisser en mélange avec la caséine produite aucune substance organique ou minérale. 